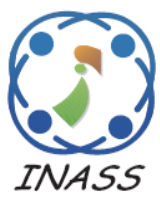

\title{
An Improved Dual Steganography Model Using Multi-pass Encryption and Quotient Value Differencing
}

\author{
Shreela Dash ${ }^{1 *} \quad$ Madhabananda Das $^{1} \quad$ Dayal Kumar Behera $^{2}$ \\ ${ }^{I}$ Kalinga Institute of Industrial Technology, Deemed to be University, Patia, Bhubaneswar, India \\ ${ }^{2}$ Silicon Institute of Technology, Bhubaneswar, India \\ * Corresponding author’s Email: shreelamamadash@gmail.com
}

\begin{abstract}
The proposed work suggests a high capacity steganography technique using dual steganography approach. It utilizes a Multi pass encryption system to scramble the mystery message before hiding. The scrambled mystery message is divided into two parts and concealed utilizing two duplicates of the same cover image. One copy of the image uses a modified Quotient value differencing approach to hide 1st part of secret message and another copy uses difference expansion method to embed the 2 nd part of secret messages. It provides more secrecy because the suggested work uses the benefits of both encryption and steganography. The investigation shows that the two stego-pictures provide high embedding capability (EC) of 1848153 bits with Peak signal to noise ratio PSNR1 of $47.2 \mathrm{~dB}$ and PSNR2 of $51.1 \mathrm{~dB}$. Further, examination with other existing methodologies shows that the suggested methodology is proven to be better in Structural similarity Index (SSIM), EC and PSNR.
\end{abstract}

Keywords: Steganography, Quotient value differencing, Difference expansion, PSNR, Multi pass encryption.

\section{Introduction}

Data communication is easier nowadays due to the huge use of the internet. This has drawn the attraction of an immense number of researchers for sharing of information. In any case, correspondence over an open channel is constantly concerned. Keeping the secrecy of data from the unauthorized users is the prime goal, cryptography and steganography assumes a predominant job to accomplish security. Steganography is an approach of communicating secret messages through the insecure channel. It is performed by embedding the mystery information inside picture, sound, and video documents [1]. In this technique the characteristics of the picture like histogram, SSIM must remain unchanged ensuing to covering the secret data in it [2]. Cryptography is a method which protects the secret information by encrypting them, so that only the authorized user can read it [4]. Steganography provides secret communication, so that no one can identify the presence of covert message and cryptography is the method used for encryption of secret message so that unauthorized user can not break it. In this paper both the cryptography and steganography approach is combined to add more secrecy to the secret communication. There exists a huge number of existing techniques in the literature but there is still scope of significant upgrades to have high Embedding limit and secrecy to mystery message.

In the proposed work, utilizing hybridization of both cryptography and steganography a high secrecy correspondence is done. The proposed strategy improves the Embedding limit by hiding the secret data in dual image utilizing QVD and Difference expansion method. Our proposed work is based on spatial domain principle as it gives emphasis to the high payload of secret data. We observed that the payload capability and the security are still less in the existing methods. So the suggested approach uses the concept of Dual steganography to increase the capability of embedding and cryptography approach for improving secrecy of hiding.

The significant enhancements of the proposed methods are given below: 
1. The proposed approach encrypts the secret message using Multi-pass encryption (MPE) technique before embedding.

2. The proposed approach effectively utilizes the two similar images efficiently for the concealing of secret information. Both the stego images are then needed to fully extract the hidden data.

3. Using QVD and DE technologies, the technique effectively hides more mysterious bits to strengthen the embedding capability.

The organization of the work is sorted out as given below. Section 2 surveys existing methodologies present in a similar area. The proposed strategy is presented in Section 3. In Section 4 the outcomes and relative analysis is introduced. At last, Section 5 describes the final outcomes.

\section{Literature survey}

Major research on image steganography has been performed over the years. These algorithms are categorized into two main classes depending on embedding method i.e. spatial domain [6, 7, and 11] and transform domain. The spatial domain embedding algorithms are used frequently because of their good concealing technique, more capacity to hide information, and simplicity of understanding [5]. LSB and pixel value difference are the most common methods in the spatial domain [2]. Some of the works in the same domain are studied in literature. The LSB matching of Mielikainen [10] is one of the typical works in this area that results in stego image with the least possible distortion of stego image. The process was nevertheless reversible. By expanding the LSB matching strategy, Lu et al. [5] uses a table of rules to preserve the quality of image. By using twin images, the $\mathrm{HC}$ of the technique has been doubled. However, Lu's technique can be further improved in terms of capacity. A novel steganographic framework depends on the techniques of PVD and modified LSB is suggested in [8]. The main contributions are to increase the amount of embedded data and with accepted distortion. However, in high EC the PSNR is nearly 35 , which can be improved. Jung et al. [9] have used two similar images for embedding to improve capacity. Mean and adjacent pixel difference are used to maintain reversibility. The Embedding capacity using dual cover image can be further improved. In [12] the authors have combined both differencing and substitution mechanisms for better performance in high capacity steganography. Firstly, the image is partitioned into independent pixel blocks of size $3 \times 3$ and on every pixel block, LSB substitution is applied on the two least significant bits and QVD is applied on the rest of the bits. The technique results in degrade of quality of stego image. H. Yuan [13] have used multiple cover images using Secret Sharing method and the suggested approach hide the encrypted bits adaptively in each cover. The suggested method results in FOBP (Fall of Boundary Problem). A method proposed by Tseng [14] hides the secret message using the edge by deciding the number of pixels to be hidden using LSB technique. It enhances the capability of hiding but the security of the technique can be further improved. Nguyen [15] has suggested adaptive LSB based MPBDH technique that uses both smooth and rough region for data hiding. To detect the complex regions of a cover image for embedding the confidential message, it uses more no. bit plane and used an adaptive complexity threshold. The embedding power and protection quality have improved dramatically relative to the previous system. Another method suggested by [16] combining directional PVD with LSB that results improvement in PSNR with high payload. F. Jafar et al. [18] suggested a RDH approach where embedding and extraction of data is performed in three consecutive steps. In the initial step, one mystery bit in every pixel is inserted utilizing four straightforward principles. The other two stages utilize the idea of prediction for inserting mystery information yet without utilizing any unpredictable indicators. However, the hiding limit can be additionally improved.

\section{Proposed work}

New steganography approaches are in current progressions and research is still going on for enhancing capacity of payload with more security and less distortion. The suggested work aims at enhancing the embedding capability and to maintain the secrecy of the communication. In the proposed approach, authors combined both the cryptography and steganography approach and get the benefits of both the techniques. To improve the secrecy of covert communication encryption technique is used and steganography methods help for secret communication. For improving the capacity of embedding algorithm, dual stego images are used. It not only improves capacity but also improves secrecy as to retrieve the secret information both the stego images are required. The proposed approach is represented in Fig. 1.

\section{Proposed dual steganography method}

1. The secret message $S$ is encrypted using Multi pass Encryption (MPE) algorithm. 


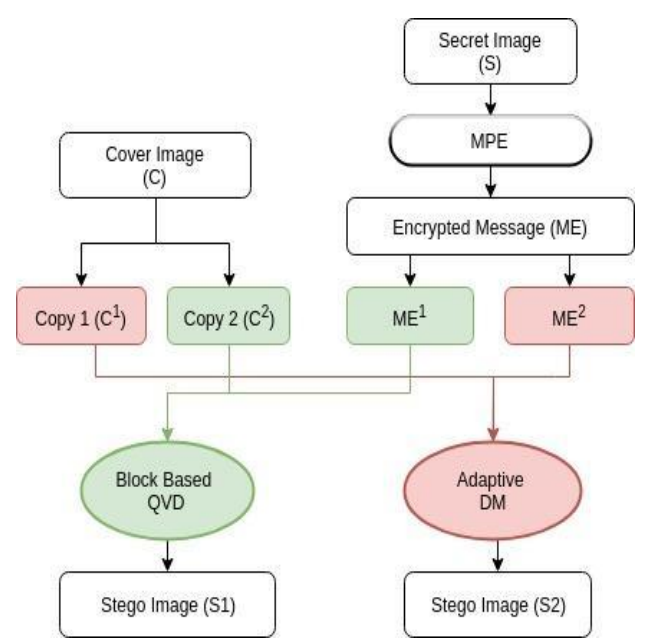

Figure. 1 The proposed dual steganography approach

2. The encrypted message $M E$ is divided into 2 parts. Odd indexed elements are kept in $\mathrm{ME}^{1}$ and even indexed elements are kept in $\mathrm{ME}^{2}$.

3. Two identical copies of the cover image $C$ are taken i.e. Copy $1 \quad\left(C^{l}\right)$ and Copy2 $\left(C^{2}\right)$ respectively.

4. $M E^{l}$ is implanted in the cover image $\mathrm{C}^{2}$ by block QVD technique and $M E^{2}$ is embedded in $C^{l}$ using Adaptive Difference Modification technique.

5. Two generated stego images $S 1$ and $S 2$ are transferred through the communication medium.

\subsection{Multi pass encryption algorithm}

The Secret message is encrypted using the following algorithm. The algorithm contains 4 steps. Input: Secret message $(S)$

Output: Encrypted message (ME)

1. In the first step each 8 bits of secret message $S$ is taken and XOR operation with 11111111 is performed.

2. The 8 bit of the secret message is divided into 4 blocks. Each block consists of 2 bits. The division is done in the following way.

$s 1=5^{\text {th }}$ and $1^{\text {st }}$ bit of each 8 bit, $s 2=6^{\text {th }}$ and $2^{\text {nd }}$ bit, $s 3=7^{\text {th }}$ and $3^{\text {rd }}$ bit. $s 4=8^{\text {th }}$ and $4^{\text {th }}$ bit. The 4 blocks are concatenated.

3. Then the shuffling of the secret bits is done using some random secret key $\mathrm{k}$. The $\mathrm{k}$ is of length 8 and it consists of 1 to 8 decimals.

4. The random secret binary key $\mathrm{k}$ is taken for encryption. If $k_{i}=1$, then XOR operation with the particular bit of secret message is done otherwise it is left unchanged.

The idea of MPE algorithm is explained with a simple example.

Consider the 8-bits of secret message $\mathrm{S}=10011011$.

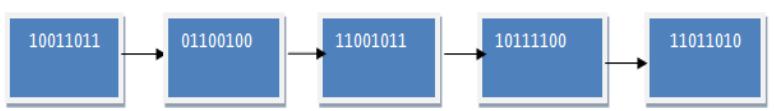

Figure. 2 Illustration of generation of encrypted secret message

As per step 1 XOR operation of the Secret message $\mathrm{S}$ with 11111111 is performed.

$S=10011011$ XOR $11111111=01100100$

As per step 2 the secret message $S$ is divided into 4 blocks i.e. $S_{1}=11, S_{2}=00, S_{3}=10, S_{4}=11$. After concatenation of the four blocks $S S=11001011$.As per step 3 the random secret key is generated for example:" $41273865 "$ ". As $I^{\text {st }}$ position of secret key is 4 so $1^{s t}$ bit of $S S$ i.e. 1 is placed in $4^{\text {th }}$ position and so on. After step 3 the generated secret message is $S S=$ 10111100 . As per step 4 random binary secret key is taken i.e. $k=01100110$. If $k_{i}=1$ XOR operation of $S S$ is performed with the corresponding bit of $k$ otherwise no operation is performed. The generated encrypted secret message is $M E=11011010$.

The illustration after each step is shown in Fig.2.

For decryption of the encrypted message, the receiver will perform the above 4 steps in reverse order.

\subsection{Embedding technique}

The cover image $C$ is copied and two same share of the cover image $C^{l}$ and $C^{2}$ are made. The scrambled secret message is partitioned into two parts. The odd indexes of EC (ME in Fig. 1) are kept in one array $E C^{l}$ and even index numbers are kept in $E C^{2}$. The $E C^{l}$ is embedded in cover image $C^{l}$ using block based QVD technique and $E C^{2}$ is embedded using adaptive DE technique. Then the dual stego images are transferred through the unsecure communication channel.

\subsubsection{Embedding using block based QVD}

Input: Identical copy of the cover image $C^{l}(I l$, $I 2, I 3, \ldots \ldots, I n)$ and the covert message $E C^{l}$.

Output: The stego image $S^{l}$

Algorithm

1. The original image $C^{l}$ is partitioned into $3 \times 3$ size blocks. From the block using Eq. (1) and Eq. (2) the high order and low order bits of each pixel of the block are separated.

$$
\begin{array}{ll}
q_{i}=I_{i} \operatorname{div} 4 & \text { for } i=1 \text { to } 9 \\
r_{i}=I_{i} \bmod 4 & \text { for } i=1 \text { to } 9
\end{array}
$$


Table 1. Range table

\begin{tabular}{|c|c|c|c|c|}
\hline $\begin{array}{c}\text { Range }\left(\mathrm{L}_{\mathrm{i}}-\right. \\
\left.\mathrm{H}_{\mathrm{i}}\right)\end{array}$ & $0-7$ & $8-15$ & $16-31$ & $32-63$ \\
\hline Capacity $\left(\mathrm{m}_{\mathrm{i}}\right)$ & 2 & 3 & 4 & 5 \\
\hline
\end{tabular}
block.

Where $q_{c}$ and $r_{c}$ are the centre pixel of each

2. Retrieve each 2 bits of secret message from $E C^{l}$ and convert it to decimal $b_{i}$. Each pixel of $r_{i}$ will be replaced with $b_{i}$.

$$
r_{i}^{\prime}=b_{i} \text { for } i=1 \text { to } 9
$$

3. For each high order pixel in $\mathrm{q}_{\mathrm{i}}$, find the difference $d_{i}$ with centre pixel $q_{c}$ using Eq. (4).

$$
d_{i}=q_{i}-q_{c} \text { for } i=1 \text { to } 9
$$

4. According to the range of distance values given in Table 1 , the capacity of each pixel to hide $\left(m_{i}\right)$ is found. $\mathrm{m}_{\mathrm{i}}$ Bits of secret data from $E C^{1}$ are transformed to decimal $b_{i}$.The modified difference $d_{i}{ }^{\prime}$ is calculated using Eq. (5).

$$
d_{i}^{\prime}=\left\{\begin{array}{c}
L_{i}+b_{i} \quad \text { if } d_{i} \geq 0 \\
-L_{i}-b_{i} \quad \text { if } d_{i}<0
\end{array}\right.
$$

5. Then the difference between modified difference and original difference is calculated using Eq. (6).

$$
k_{i}=d_{i}^{\prime}-d_{i}
$$

6. Then $\mathrm{q}_{\mathrm{i}}^{\prime}$ is calculated using using Eq. (7)

$$
q_{i}^{\prime}=q_{i}+k_{i}
$$

7. The stego pixels are calculated using Eq. (8)

$$
S^{1}(i)=q_{i}{ }^{\prime} \times 4+r_{i}^{\prime} \text { for } i=1 \text { to } 9
$$

\subsubsection{Embedding using adaptive DE}

Input: Identical copy of the cover image $C^{2}(\mathrm{~J} 1$, $\mathrm{J} 2, \mathrm{~J} 3, \ldots \ldots, \mathrm{Jn})$ and the covert message $E C^{2}$.

Output: Stego image $S^{2}$.

Algorithm

1. Divide $C^{2}$ into independent blocks of size $3 \times 3$.

2. Each 3 bits of secret message from $E C^{2}$ is converted to decimal $b_{i}$. Each pixel is converted to binary and the last 3 bits are converted to decimal $p_{i}$.

3. Find the difference $d_{i}$ using Eq. (12).

$$
d_{i}=p_{i}-b_{i}
$$

4. Modified pixels ${j_{i}}^{\prime},{j_{i}}^{\prime \prime},{j_{i}}^{\prime \prime \prime}$ are found using Eqs. (13), (14), and (15) respectively.

$$
\begin{gathered}
j_{i}{ }^{\prime}=j_{i}-d_{i} \\
j_{i}{ }^{\prime \prime}=j_{i}-2^{3} \\
j_{i}{ }^{\prime \prime \prime}=j_{i}+2^{3}
\end{gathered}
$$

5. The stego-pixel $S^{2}(\mathrm{i})$ is calculated using Eq. (16).

$$
S^{2}(\mathrm{i})=\min \left(j_{i}{ }^{\prime}, j_{i}{ }^{\prime \prime}, j_{i}{ }^{\prime \prime \prime}\right)
$$

\subsection{Extraction technique}

The dual stego images $S^{1}$ and $S^{2}$ are transferred through the communication medium. From both the stego images the covert bits are generated to get the scrambled message. The reverse steps of multi pass encryption are performed to generate original secret message. .

\subsubsection{Extraction algorithm for QVD}

Input: Stego-image received through communication channel $S^{1}$.

Output: The encrypted message $E C^{1}$.

Algorithm

1. The stego-image $S^{1}$ is split into $3 \times 3$ independent blocks. Then the high-order and low-order bits are generated using Eq. (9) and (10), respectively.

$$
\begin{aligned}
& \mathrm{q}_{\mathrm{i}}=S^{1}(\mathrm{i}) \operatorname{div} 4 \text { for } \mathrm{i}=1 \text { to } 9 \\
& \mathrm{r}_{\mathrm{i}}=S^{1}(\mathrm{i}) \bmod 4 \text { for } \mathrm{i}=1 \text { to } 9
\end{aligned}
$$

2. Each pixel in $r_{i}$ is transformed to binary and retrieve the last 2 bits of each pixel add it to $\mathrm{EC}^{1}$.

3. For each block $q_{i}$, the difference of each quotient pixel with the centre pixel is calculated using Eq. (11).

$$
d_{i}=q_{i}-q_{c}
$$


4. For each $d_{i}$ we can generate $\mathrm{L}_{\mathrm{i}}$ and capacity $\mathrm{m}_{\mathrm{i}}$ using the Table $1 . b_{i}{ }^{\prime}$ is calculated and is transformed to $\mathrm{m}_{\mathrm{i}}$ binary bits and are appended to $E C^{1}$

\subsubsection{Extraction algorithm for adaptive $D E$}

Input: Stego-image received through communication channel $S^{2}$.

Output: The encrypted message $E C^{2}$.

Algorithm

1. The stego-image $S^{2}$ is split into $3 \times 3$ independent blocks.

2. Convert pixels of each block to binary and retrieve 3 LSB bits.

3. Append these bits to the extracted bits $E C^{2}$.

4. Then $E C^{l}$ and $E C^{2}$ are arranged in an odd and even position sequentially to generate the encrypted message $E C$.

\subsection{Illustration of embedding and extraction algorithm}

To explain the embedding and extraction algorithm, we use a $3 \times 3$ block given in Fig. 3 . The encrypted secret bit $E C$ is divided into 2 parts

$E C^{1}=100110110101001101110011011$

$E C^{2}=101110010100011100100101000$

\begin{tabular}{|l|l|l|}
\hline 170 & 160 & 149 \\
\hline 171 & 158 & 173 \\
\hline 173 & 173 & 170 \\
\hline
\end{tabular}$\quad$\begin{tabular}{ll|l|l|}
\hline 42 & 40 & 37 \\
\hline 42 & 39 & 43 \\
\hline 43 & 43 & 42 \\
\hline
\end{tabular}$\quad$\begin{tabular}{ll|l|l|}
\hline 2 & 0 & 1 \\
\hline 3 & 2 & 1 \\
\hline 1 & 1 & 2 \\
\hline
\end{tabular}

Figure. 3 Original block, high order and low order pixel

\begin{tabular}{|l|l|l|l|l|l|}
\hline 42 & 39 & 42 \\
\hline 41 & 39 & 41 \\
\hline 40 & 42 & 41 \\
\hline 2 & 1 & 2 \\
\hline 3 & 1 & 1 \\
0 & 3 & 1 \\
\hline
\end{tabular} \mid \begin{tabular}{|l|l|l|l|}
170 & 157 & 170 \\
\hline 171 & 157 & 165 \\
\hline 160 & 171 & 165 \\
\hline
\end{tabular}

Figure. 4 High order, Low order pixel values and generated stego block

\begin{tabular}{|l|l|l|}
\hline 170 & 160 & 149 \\
\hline 171 & 158 & 173 \\
\hline 173 & 173 & 170 \\
\hline
\end{tabular}

\begin{tabular}{|l|l|l|}
\hline 162 & 154 & 152 \\
\hline 170 & 155 & 175 \\
\hline 173 & 173 & 170 \\
\hline
\end{tabular}

Figure. 5 Original block and corresponding stego block
As per the algorithm the high-order and low-order bits are calculated as per Eqs. (1) and (2) which is given in Fig. 3.

Two secret bits from $E C^{1}$ are converted to decimal and set as values in $r_{i}{ }^{\prime}$. Then as per Eq. (4) the difference between $q_{i}$ and $q_{c}$ is calculated. So, $d_{1}=3, d_{2}=1, d_{3}=-2, d_{4}=3, d_{5}=4, d_{6}=4, d_{7}=4$ and $d_{8}=3$. Now from Table 1 we found the difference di is within the range 0 to 7 , Lower index $L_{i}=0$ and capacity $m_{i}=2$. As the hiding capacity is 2,2 bits of secret data are converted to decimal i.e. $b_{1}=3, b_{2}=0$, $b_{3}=3, b_{4}=1, b_{5}=2, b_{6}=1, b_{7}=3, b_{8}=2$. The modified difference $d_{i}{ }^{\prime}$ is calculated using $L_{i}$ and $b_{i}$. So $d_{1}{ }^{\prime}=3$, $d_{2}{ }^{\prime}=0, d_{3}{ }^{\prime}=3, d_{4}{ }^{\prime}=1, d_{5}{ }^{\prime}=2, d_{6}{ }^{\prime}=1, d_{7}{ }^{\prime}=3, d_{8}{ }^{\prime}=2$. Then difference between di and $\mathrm{d}_{\mathrm{i}}{ }^{\prime}$ calculated and kept in ki. So $k_{1}=0, k_{2}=-1, k_{3}=5, k_{4}=-2, k_{5}=-2, k_{6}=-3$, $k_{7}=-1$ and $k_{8}=-1$. The modified $q_{i}{ }^{\prime}$ is calculated as per Eq. (7). The values are $q_{1}{ }^{\prime}=42, q_{2}{ }^{\prime}=39, q_{3}{ }^{\prime}=42$, $q_{4}{ }^{\prime}=41, q_{5}{ }^{\prime}=41, q_{6}{ }^{\prime}=40, q_{7}{ }^{\prime}=42, q_{8}{ }^{\prime}=41$. As per Eq. (8) the calculated stego pixels along with high order and low order bits are shown in Fig. 4.

After getting the first stego block, the second pair of secret bits $E C^{2}$ is hidden inside the original block using Adaptive DE algorithm. Here 3 bits of secret message are converted to decimal and kept in $b_{i}$. So, $b_{1}=5, b_{2}=6, b_{3}=2, b_{4}=4, b_{5}=3, b_{6}=4, b_{7}=4, b_{8}=5$, $b_{9}=0$. Then the last 3 bits of each pixel in the block are converted to decimal and kept in $p_{i}$. So $p_{1}=2, p_{2}=0$, $p_{3}=5, p_{4}=3, p_{5}=6, p_{6}=5, p_{7}=5, p_{8}=5, p_{9}=2$. The $d_{i}$ is calculated as per Eq. (9). So $d_{1}=-3, d_{2}=-6, d_{3}=3, d_{4}=$ $-1, d_{5}=3, d_{6}=2, d_{7}=1, d_{8}=0$ and $d_{9}=2$. The generated stego block is calculated and given in Fig. 5 .

The receiver will take out the mystery bits from the generated stego images $S^{1}$ and $S^{2}$. From $S^{1}$ the high order bits $\left(q_{i}{ }^{\prime}\right)$ and low order bits $\left(r_{i}{ }^{\prime}\right)$ are generated. Each pixel $r_{i}{ }^{\prime}$ of the block is converted to 2 bits binary and added to the 1D array $E C^{l}$. Then from $\left(q_{i}{ }^{\prime}\right)$ the difference $d_{i}{ }^{\prime}$ is calculated. The calculated values are $d_{1}{ }^{\prime}=3, d_{2}{ }^{\prime}=0, d_{3}{ }^{\prime}=3, d_{4}{ }^{\prime}=1$, $d_{5}{ }^{\prime}=2, d_{6}{ }^{\prime}=1, d_{7}{ }^{\prime}=3, d_{8}{ }^{\prime}=2$. From Table (1) the range is found. Here $L_{i}=0$ and $m_{i}=2$. So the values are $b_{1}=3, b_{2}=0, b_{3}=3, b_{4}=1, b_{5}=2, b_{6}=1, b_{7}=3, b_{8}=2$ and each $b_{i}$ is converted to 2 bit binary and appended to $E C^{l}$. Similarly from $S^{2}$ the last 3 binary bits of each pixel are generated and appended to $E C^{2}$. Now $E C^{l}$ and $E C^{2}$ are combined to generate the encrypted secret message EC.

\section{Result analysis}

The experiment was conducted utilizing MATLAB R-2017. The original images were chosen 

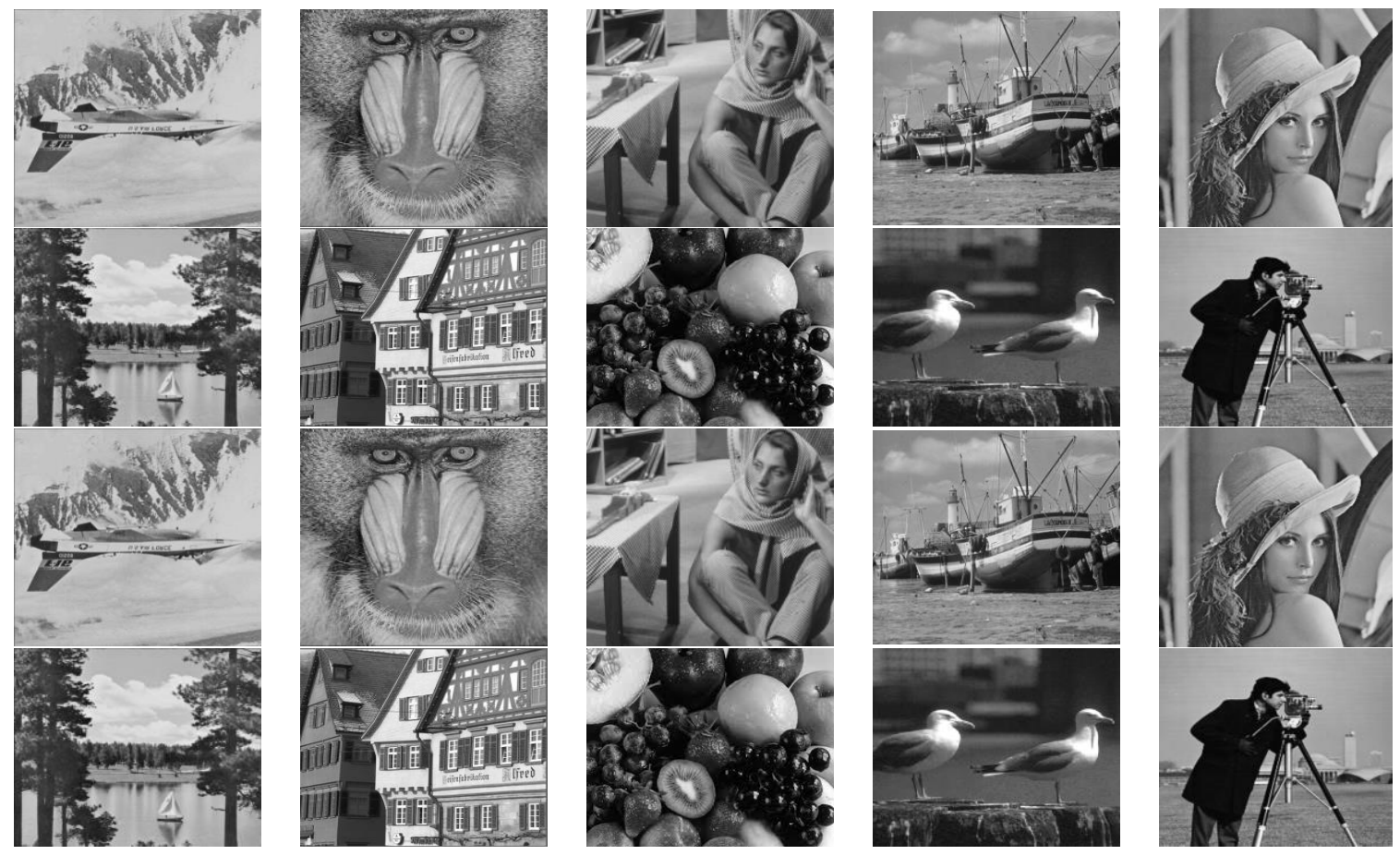

Figure. 6 Cover image and corresponding stego image

Table 2. Analysis of Proposed method and Jung's [9] method

\begin{tabular}{|c|c|c|c|c|c|}
\hline \multirow{2}{*}{$\begin{array}{c}\text { Test Image } \\
(512 \times 512)\end{array}$} & \multicolumn{5}{|c|}{ Proposed Technique } \\
\cline { 2 - 6 } & EC & PSNR1 & PSNR2 & SSIM1 & SSIM2 \\
\hline Airplane & $18,21,220$ & 48.3 & 52.2 & 0.9987 & 0.9989 \\
\hline Boat & $18,67,436$ & 46.6 & 52.0 & 0.9992 & 0.9986 \\
\hline House & $18,67,436$ & 46.6 & 49.9 & 0.9989 & 0.9997 \\
\hline Scenery & $18,50,455$ & 46.9 & 51.0 & 0.9986 & 0.9992 \\
\hline Lena & $18,60,400$ & 47.1 & 49.9 & 0.9987 & 0.9988 \\
\hline Baboon & $18,21,220$ & 48.3 & 52.1 & 0.9978 & 0.9980 \\
\hline Zelda & $18,67,436$ & 46.8 & 50.2 & 0.9991 & 0.9993 \\
\hline Bird & $18,60,670$ & 47.3 & 50.0 & 0.9987 & 0.9987 \\
\hline Cameraman & $18,35,008$ & 48.1 & 51.8 & 0.9982 & 0.9984 \\
\hline Fruit & $18,30,250$ & 48.2 & 51.9 & 0.9983 & 0.9983 \\
\hline Average & $18,48,153$ & 47.2 & 51.1 & 0.9986 & 0.9988 \\
\hline
\end{tabular}

\begin{tabular}{|c|c|c|c|c|c|}
\hline \multirow{2}{*}{$\begin{array}{c}\text { Test Image } \\
(512 \times 512)\end{array}$} & EC & PSNR1 & PSNR2 & SSIM1 & SSIM2 \\
\cline { 2 - 6 } & $7,81,964$ & 45.5 & 47.4 & 0.9857 & 0.9865 \\
\hline Airplane & $7,86,097$ & 45.4 & 47.5 & 0.9906 & 0.9886 \\
\hline Boat & $7,79,948$ & 45.5 & 47.4 & 0.9922 & 0.9902 \\
\hline House & $7,70,943$ & 46.9 & 51.0 & 0.9887 & 0.9880 \\
\hline Scenery & $7,73,666$ & 45.4 & 47.4 & 0.9860 & 0.9885 \\
\hline Lena & $7,72,544$ & 45.5 & 47.5 & 0.9878 & 0.9980 \\
\hline Baboon & $7,85,008$ & 45.3 & 47.4 & 0.9880 & 0.9850 \\
\hline Zelda & $7,65,090$ & 45.5 & 47.1 & 0.9887 & 0.9967 \\
\hline Bird & $7,65,312$ & 45.5 & 47.2 & 0.9880 & 0.9859 \\
\hline Cameraman & $7,60,506$ & 45.5 & 47.3 & 0.9883 & 0.9873 \\
\hline Fruit & $7,74,107$ & 45.6 & 47.7 & 0.9884 & 0.9895 \\
\hline Average & & & & & \\
\hline
\end{tabular}

from USC-SIPI image databases with a size of $512 \times 512$ pixels [17]. The original and stego images are given in Fig. 6. The suggested technique is implemented and compared with the existing methodologies in the same domain [3,5,9]. The work used the (PSNR) to measure the pixel gap between stego images and the original image. 
Table 3. Result of Lu's [5] and Qin's [3] method

\begin{tabular}{|c|c|c|c|c|c|}
\hline \multirow{2}{*}{$\begin{array}{l}\text { Test Image } \\
(512 \times 512)\end{array}$} & \multicolumn{5}{|c|}{ LU's [5] } \\
\hline & $\mathrm{EC}$ & PSNR1 & PSNR2 & SSIM1 & SSIM2 \\
\hline Airplane & $5,24,288$ & 49.11 & 49.09 & 0.9983 & 0.9985 \\
\hline Boat & $5,24,208$ & 49.14 & 49.09 & 0.9989 & 0.9986 \\
\hline House & $5,24,996$ & 49.15 & 48.86 & 0.9981 & 0.9982 \\
\hline Scenery & $5,24,288$ & 49.11 & 49.1 & 0.9985 & 0.9987 \\
\hline Lena & $5,24,288$ & 49.13 & 49.12 & 0.9987 & 0.9988 \\
\hline Baboon & $5,22,240$ & 49.14 & 49.09 & 0.9983 & 0.9985 \\
\hline Zelda & $5,24,996$ & 49.13 & 49.12 & 0.9991 & 0.9993 \\
\hline Bird & $5,24,280$ & 49.11 & 49.14 & 0.9967 & 0.9972 \\
\hline Cameraman & $5,22,244$ & 49.03 & 49.11 & 0.9980 & 0.9982 \\
\hline Fruit & $5,22,240$ & 49.13 & 49.09 & 0.9983 & 0.9984 \\
\hline Average & $5,23,806$ & 49.11 & 49.08 & 0.9983 & 0.9984 \\
\hline
\end{tabular}

\begin{tabular}{|c|c|c|c|c|c|}
\hline \multirow{2}{*}{$\begin{array}{c}\text { Test Image } \\
(512 \times 512)\end{array}$} & EC & PSNR1 & PSNR2 & SSIM1 & SSIM2 \\
\cline { 2 - 6 } & $5,57,339$ & 41.55 & 52.12 & 0.9867 & 0.9885 \\
\hline Airplane & $5,57,194$ & 41.57 & 52.11 & 0.9866 & 0.9886 \\
\hline Boat & $5,57,948$ & 41.48 & 51.91 & 0.9867 & 0.9882 \\
\hline House & $5,57,564$ & 41.58 & 52 & 0.9870 & 0.9880 \\
\hline Scenery & $5,57,552$ & 41.58 & 52.11 & 0.9867 & 0.9884 \\
\hline Lena & $5,57,264$ & 41.34 & 52.12 & 0.9863 & 0.9878 \\
\hline Baboon & $5,57,948$ & 41.48 & 52.1 & 0.9867 & 0.9881 \\
\hline Zelda & $5,57,552$ & 41.48 & 52.11 & 0.9871 & 0.9882 \\
\hline Bird & $5,57,948$ & 41.11 & 51.91 & 0.9863 & 0.9879 \\
\hline Cameraman & $5,57,260$ & 41.19 & 52.15 & 0.9870 & 0.9878 \\
\hline Fruit & $5,57,556$ & 41.43 & 52.06 & 0.9867 & 0.9882 \\
\hline Average & & & & & \\
\hline
\end{tabular}

Boat
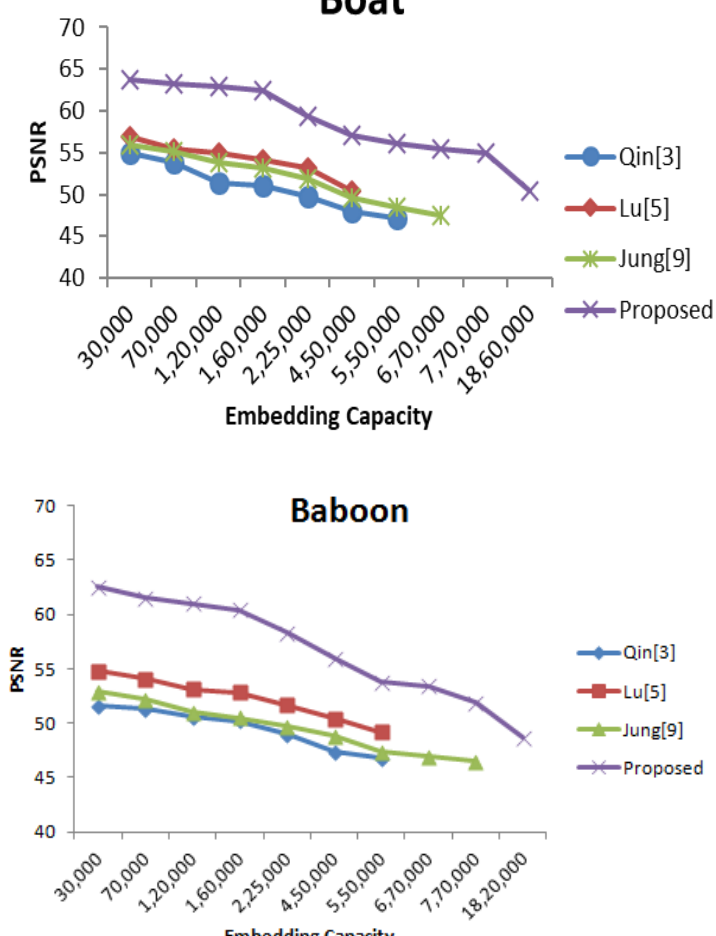

Lena

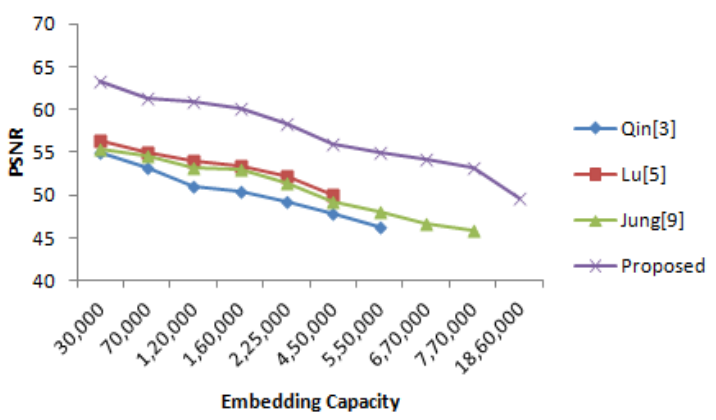

House

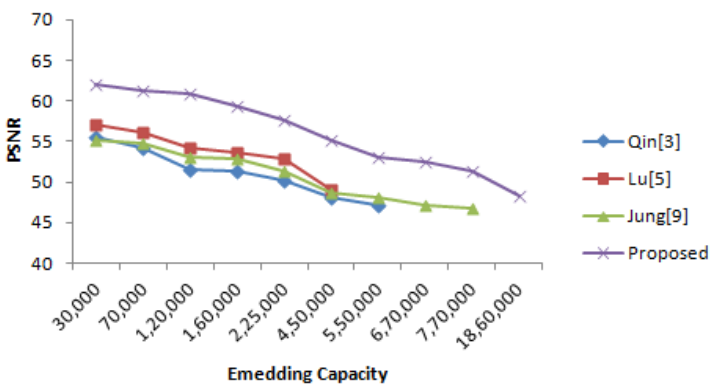

Figure. 7 EC vs PSNR of different test images 


$$
P S N R=10 \log _{10} \frac{M a x^{2}}{\frac{1}{m \times n} \sum_{i=1}^{n} \sum_{j=1}^{n}\left(C_{i j}-S_{i j}\right)^{2}}
$$

Here Max is used to denote the maximum intensity value in the image. $c_{i j}$ and $s_{i j}$ represent the corresponding pixels of the Test and Stego image respectively. The performance of the algorithm is also evaluated based on different parameters like Embedding capacity and Structural similarity Index. SSIM value close to 1 increases stego-image efficiency [2]. The SSIM can be evaluated using Eq. (18).

$$
S S I M=\frac{\left(2 \bar{p} q+C_{1}\right)\left(2 \sigma_{p q}+C_{2}\right)}{\left(\overline{p^{2}}+\overline{q^{2}}+C_{1}\right)\left(\sigma_{p}^{2}+\sigma_{q}^{2}+C_{2}\right)}
$$

$\overline{\mathrm{p}}, \overline{\mathrm{p}^{2}}, \sigma_{\mathrm{p}}{ }^{2}$ and $\overline{\mathrm{q}}, \overline{\mathrm{q}^{2}}, \sigma_{\mathrm{q}}{ }^{2}$ reprsent the mean, variance and the standard deviation of the the original and stego image respectively. Likewise $2 \sigma_{\mathrm{pq}}$ is the covariance and the two constants are $c_{1}$ and $c_{2}$.

The comparison of the stego image in the suggested approach with the existing Jung's [9] technique is shown in Table 2. Here PSNR1, PSNR2, SSIM1 and SSIM2 represent the PSNR and SSIM value of the 1st stego-image, 2nd stego-image respectively. Table 2 shows SSIM value is nearly equal to 1 for all the test images taken. The PSNR, Embedding capacity and SSIM of the existing approach Qin's [3] and Lu's [5] is given in Tables 3. The result analysis shows that the embedding capability of the suggested technique is much more higher than the other existing techniques of same domain. For ex: Boat image has hiding capacity of 18 , 67,436 with PSNR1 46.6 and PSNR2 is 50 in proposed method where as in Jung [9] the hiding capacity is 7,86,097 with PSNR1 is 45.5 and PSNR2 is 47.4. In $\mathrm{Lu}$ [5] the hiding capacity is 5,24,208 with PSNR value 49 and Qin [3] provides 5,57,194 hiding capacity with PSNR1 41.57 and PSNR2 52.11. The average embedding capability of the proposed method is $18,48,153$ which is much more than the other existing methodologies shown in Table 2.

Similarly SSIM1 and SSIM2 of images shown in Table 2 and Table 3 also prove that SSIM value of the suggested approach is higher than the existing methods. Average SSIM1 of proposed method is 0.9986 and PSNR2 is 0.9988 which is nearly equal to 1 which shows that the image quality is better.

Fig. 7 shows the relation of embedding capability with PSNR for 4 different images Lena, Boat, House and Baboon. The result shows that with increasing embedding capacity PSNR decreases. It also shows with embedding capacity 30000 bits the proposedmethod results in PSNR value more than 60 whereas Jung's [9], Lu's [5] and Qin's [3] exhibits PSNR value more than 50 .

\section{Conclusions}

A high capacity information hiding technique is proposed using Multi pass encryption, QVD and adaptive DE technique. The secret message is scrambled using MPE technique to use the benefit of cryptography and providing more secrecy. The dual images of same cover image is obtained and used to conceal the encrypted secret message. QVD technique is used to hide the part of secret message in $1^{\text {st }}$ cover image and adaptive DE technique is used to embed the rest part in $2^{\text {nd }}$ cover image. The proposed method provides the embedding capacity of 18,48,153 with PSNR1 47.2 and PSNR2 52.1 in average for the Test images. The comparison is also done with the existing techniques of same domain and the result shows that the proposed technique has improved EC, PSNR and SSIM. In the future the capacity can be further improved by identifying the features of each block to generate the capacity of hiding. The proposed work will be further extended to validate for unauthorized attack.

\section{Conflicts of Interest}

The authors declare no conflict of interest.

\section{Author Contributions}

Conceptualization, methodology, software, investigation, validation, writing - original draft preparation, writing and editing, S. Dash; investigation, review, supervision, M.N. Das; resources, formal analysis, writing-review and editing, D.K. Behera.

\section{References}

[1] I. J. Kadhim, P. Premaratne, P. J. Vial, and B. Halloran, "Comprehensive survey of image steganography: Techniques, Evaluations, and trends in future research", Neuro computing, Vol. 335, pp. 299-326, 2019

[2] M. Hussain, A. W. A. Wahab, Y. I. B. Idris, A. T. Ho, and K. H. Jung, "Image steganography in spatial domain: A survey", Signal Processing: Image Communication, Vol. 65, pp. 46-66, 2018.

[3] C. Qin, C. Chang, and T. Hsu, "Reversible data hiding scheme based on exploiting modification direction with two steganographic images", Multimed Tools Appl, Vol. 74, pp. 5861-5872, 2015.

[4] S. Dash, M. N Das, and M. Das, "Secured Image Transmission through Region-Based 
Steganography Using Chaotic Encryption, Computational Intelligence in Data Mining", Advances in Intelligent Systems and Computing, Vol. 711. Springer, 2018

[5] T. Lu, C. Tseng, and J. Wu, "Dual imagingbased reversible hiding technique using LSB matching", Signal Processing, Vol. 108, pp. 7789, 2015,

[6] A. Zakaria, M. Hussain, A. Wahab, M. Idris, N. Abdullah, and K.-H. Jung, "High-Capacity Image Steganography with Minimum Modified Bits Based on Data Mapping and LSB Substitution", Applied Sciences, Vol. 8, No. 11, 2018.

[7] J. Wang, J. Ni, X. Zhang, and Y. Q. Shi, "Rate and Distortion Optimization for Reversible Data Hiding Using Multiple Histogram Shifting", IEEE Transactions on Cybernetics, Vol. 47, No. 2, pp. 315-326, 2017.

[8] K. A. Darabkh, A. K. Al-Dhamari, and I. F. Jafar, "A new steganographic algorithm based on multi directional PVD and modified LSB", Journal of Information Technology and Control, Vol. 46, No.1, pp. 16-36, 2017.

[9] K. H. Jung, "Dual image based reversible data hiding method using neighbouring pixel value differencing", The Imaging Science Journal, Vol. 63, No. 7, pp. 398-407, 2015.

[10] J. Mielikainen, "LSB matching revisited", IEEE Signal Processing Letters, Vol. 13, No. 5, pp. 285-287, 2006.

[11] T.-C. Lu, C.-Y. Tseng, S.-W. Huang, and T. Nhan, "Pixel-Value-Ordering based Reversible Information Hiding Scheme with Self-Adaptive Threshold Strategy", Symmetry, Vol. 10, No. 12, p. 764, 2018.

[12] G. Swain, "Very High Capacity Image Steganography Technique Using Quotient Value Differencing and LSB Substitution", Arabian Journal for Science and Engineering, Vol. 44, No. 4, pp. 2995-3004, 2019.

[13] H. D. Yuan, "Secret sharing with multi-cover adaptive steganography", Information Sciences an International Journal., Vol. 254. pp. 197-212, 2014.

[14] H. Tseng and H. Leng, "High-payload blockbased data hiding scheme using hybrid edge detector with minimal distortion", in IET Image Processing, Vol. 8, No. 11, pp. 647-654, 2014.

[15] T. D. Nguyen, S. Arch-int, and N. Arch-int "An adaptive multi bit-plane image steganography using block data-hiding", Multimedia Tools Appl, Vol. 75, pp. 8319-8345,2016.
[16] G. Swain, "A steganographic method combining LSB substitution and PVD in a block", Procedia Comput. Sci., Vol. 85, pp. 39-44, 2016.

[17] USC-SIPI Image Database. [Online]. Available: http://sipi.usc.edu/database/database.php? volume $=$ misc. Accessed 192019.

[18] F. Jafar, K. A. Darabkh, R. T. Al-Zubi, and R. R. Saifan, "An efficient reversible data hiding algorithm using two steganographic images", Signal Processing, Vol. 128, pp. 98-109, 2016. 